\title{
The temperature outside a heated plume
}

\author{
A.M. Watts
}

\begin{abstract}
A calculation is made of the temperature distribution outside the laminar part of the plume produced by a small source of heat in an infinite fluid. It is shown in particular that the temperature variation is not exponentially small outside the plume, as might be expected in such a problem of boundary layer type.
\end{abstract}

\section{Introduction}

The structure of the plume above a small source of heat has been considered by Yih [4], Mahony [2], Batchelor [1] and many others. For moderate distances above the source, that is for distances large compared with the source size and the diffusion length but less than the critical distance at which turbulence develops, the velocity and temperature distributions are given by a similarity solution. Yih has found explicit expressions for the similarity solution for two particular values of the Prandtl number.

Further interest in the problem comes from the algebraic decay of temperature and vorticity at the outer edge of the plume. It is shown here that the temperature has an algebraic approach to the ambient value in all directions, even outside the plume, as the distance from the source increases. This is in contrast to the behaviour in the plane flow produced by a line source of heat and in almost any boundary layer problem that has been worked out. 


\section{Plume solution}

We use the Boussinesq equations. In dimensionless form with axial symmetry and no swirl they are

$$
\begin{aligned}
& u \frac{\partial u}{\partial x}+v \frac{\partial u}{\partial r}=-\frac{\partial p}{\partial x}+\sigma \nabla^{2} u+T \\
& u \frac{\partial v}{\partial x}+v \frac{\partial v}{\partial r}=-\frac{\partial p}{\partial r}+\sigma\left(\nabla^{2} v-\frac{u}{T^{2}}\right), \\
& u \frac{\partial T}{\partial x}+v \frac{\partial T}{\partial r}=\nabla^{2} T
\end{aligned}
$$

where cylindrical polar coordinates and components are used, $\sigma$ is the Prandtl number and

$$
\nabla^{2} \equiv \frac{1}{r} \frac{\partial}{\partial r}\left(r \frac{\partial}{\partial r}\right)+\frac{\partial^{2}}{\partial x^{2}} .
$$

In the plume far above the source, the approximate equations are

$$
\begin{aligned}
u \frac{\partial u}{\partial x}+v \frac{\partial u}{\partial r} & =\frac{\sigma}{r} \frac{\partial}{\partial r}\left(r \frac{\partial u}{\partial r}\right)+T, \\
u \frac{\partial T}{\partial x}+v \frac{\partial T}{\partial r} & =\frac{1}{r} \frac{\partial}{\partial r}\left(r \frac{\partial T}{\partial r}\right), \\
p & =\text { const },
\end{aligned}
$$

and the heat flux condition is

$$
2 \pi \int_{0}^{\infty} u T r d r=1,
$$

where the temperature $T$ is measured relative to the temperature at infinity.

The solution of the plume equations is of the form

$$
\begin{aligned}
& T=x^{-1} g\left(\frac{x}{x^{1 / 2}}\right), \\
& \psi=x f\left(\frac{r}{x^{1 / 2}}\right),
\end{aligned}
$$

where $\psi$ is the Stokes stream function. The upward volume flux in the plume is $2 \pi A x$, where $A=f(\infty)$, so that there is an entrainment by the 
plume of uniform strength $2 \pi A$ per unit length.

For $\sigma$ equal to 1 and 2 , the solutions derived by $Y$ ih are given explicitly by

$$
\begin{aligned}
& g(\eta)=\frac{C}{\left(B+\eta^{2}\right)^{A}}, \\
& f(n)=\frac{2 A \eta^{2}}{B+\eta^{2}},
\end{aligned}
$$

where for $\sigma=1$ :

$$
A=3, C=96 B,
$$

and for $\sigma=2$ :

$$
A=4, C=256 B^{2} \text {. }
$$

In both cases

$$
\frac{B^{A}}{C}=\frac{4 \pi A}{A+1}
$$

From (10) we have for large $n$

$$
g(\eta) \sim \frac{C}{\eta^{2 A}},
$$

and it can be shown that $g$ has this asymptotic form for all Prandtl numbers, where the constant $A$ is defined to be $f(\infty)$ as above.

\section{Outer region}

The outer flow is, to a first approximation, irrotational and at a constant temperature. It is determined by the entrainment into the plume and is given by the stream function

$$
\psi=\frac{1}{2} A\left(x+\sqrt{x^{2}+r^{2}}\right),
$$

as derived by Spunde [3]. The stream lines are parabolas with axes coincident with the axis of symmetry, so that the flow into the plume is normal to it.

To determine the eventual form of decay of the temperature, solutions of (3) are found with $u$ and $v$ derived from the stream function in (14). 
We use orthogonal coordinates $\alpha, \beta$ given by

$$
\begin{aligned}
& \alpha=x+\sqrt{x^{2}+r^{2}}, \\
& \beta=-x+\sqrt{x^{2}+r^{2}},
\end{aligned}
$$

and (3) becomes

$$
\frac{\partial}{\partial \alpha}\left(\alpha \frac{\partial T}{\partial \alpha}\right)+\frac{\partial}{\partial \beta}\left(\beta \frac{\partial T}{\partial \beta}+A T\right)=0
$$

The solution is of the form

$$
T=\int_{0}^{\infty} F(k) J_{0}(k \xi) n^{-A_{K}}(2 k n) d k
$$

where

$$
\xi=\alpha^{\frac{1}{2}}, \eta=\beta^{\frac{1}{2}} .
$$

This solution is required to match the plume solution, given in part by (8), (13). For large $x$,

$$
\begin{aligned}
& \alpha \sim 2 x, \quad \xi \sim(2 x)^{\frac{1}{2}}, \\
& \beta \sim \frac{x^{2}}{2 x}, \quad \eta \sim \frac{x}{(2 x)^{1 / 2}},
\end{aligned}
$$

and the principal contribution to the integral in (17) comes from small values of $k$. With

$$
K_{A}(2 k n) \sim(2 k n)^{-A} \text { for } k n \rightarrow 0,
$$

the asymptotic form of $T$ is

$$
T \sim n^{-2 A} \int_{0}^{\infty}(2 k)^{-A} F(k) J_{0}(k \xi) d k,
$$

which agrees with (13). Because of (8), we also require

$$
\int_{0}^{\infty}(2 k)^{-A} F(k) J_{0}(k \xi) d k \sim \frac{\text { const }}{\xi^{2}},
$$

which implies 


$$
(2 k)^{-A} F(k) \sim \text { const } k \log k \text {, for } k \rightarrow 0 \text {. }
$$

We then have for large $\frac{\eta}{\xi}$ and $n$

$$
T \sim \eta^{-A} \int_{0}^{\infty} F(k) K_{A}(2 k n) d k
$$

$$
\sim n^{-A} \int_{0}^{\infty} C k^{A+1} \log k k_{A}(2 k n) d k
$$

$\sim c n^{-2} \log n$,

so that $T$ does not decay exponentially at any stage.

To continue the asymptotic expansion of the plume solution to more terms, boundary conditions would have to be supplied from the outer solution given by (17). As well as allowing for the difference between $\eta$ and $\frac{r}{(2 x)^{1 / 2}}$, which is of order $x^{-1} \eta^{2}$, that is $2 \xi^{-2} \eta^{2}$, we would need to extend the asymptotic from (18) by

$$
K_{A}(2 k n) \sim(2 k n)^{-A}\left(1+a(2 k n)^{2}+\ldots\right) .
$$

The extra factor $k^{2}$ in this extra term would give an extra factor $\xi^{-2}$ in the next term in the asymptotic expansion for $T$, so that we would now have

$$
T \sim \frac{\text { const }}{\eta^{A} \xi^{2}}\left(1+\text { const }\left(\frac{\eta}{\xi}\right)^{2}+\ldots\right)
$$

which is consistent with an expansion of the plume solution in inverse powers of $x$.

\section{References}

[1] G.K. Batchelor, "Heat convection and buoyancy effects in fluids", Quart. J. Roy. Met. Soc. 80 (1954), 339-358.

[2] J.J. Mahony, "Heat transfer at small Grashof numbers", Proc. Roy. Soc. London A $238(1956 / 1957), 412-423$. 
[3.] Walter Guntis Spunde, "Laminar free convection from a point heat source", (M.Sc. Thesis, University of Queensland, 1967/8).

[4] Chia-Shun Yih, "Free convection due to a point source of heat", Proc. First U.S. Nat. Congr. Applied Mechanics, IZZinois Inst. Technology, Chicago, 1951, 941-947 (Amer. Soc. Mech. Eng., New York, 1952).

University of Queensland,

St Lucia,

QueensIand;

and

University of Western Ontario,

London,

Ontario, Canada. 